\title{
INTRODUCTION TO METRIC-PRESERVING FUNCTIONS
}

\section{Paul Corazza}

Amer. Math. Monthly, Volume 104, Number 4, April 1999, pages 309-323

1. Introduction. Under what conditions on a function $f:[0, \infty) \rightarrow[0, \infty)$ is it the case that for each metric space $(X, d), f \circ d$ is still a metric, and, moreover, $d$ and $f \circ d$ are equivalent metrics?

It is well-known that for any metric $d, d /(1+d)$ is a (bounded) metric that is equivalent to $d$. On the other hand, $d /\left(1+d^{2}\right)$ need not be a metric; see Proposition 2.7. We call $f:[0, \infty) \rightarrow[0, \infty)$ metric-preserving (respectively, strongly metric-preserving) if for all metric spaces $(X, d), f \circ d$ is a metric (respectively, is a metric that is topologically equivalent to $d$ ).

Although the first reference in the literature to the notion of metric-preserving functions seems to be [19], the first detailed study of these functions was by Sreenivasan in 1947 [16]. Kelley's classic text in general topology mentions some of Sreenivasan's results in an exercise [14, p. 131]:

Exercise (Kelley). Suppose $f:[0, \infty) \rightarrow[0, \infty)$ is continuous, nondecreasing, and subadditive $(f(x+y) \leq f(x)+f(y)$ for all $x, y)$. Suppose also that $f^{-1}(0)=\{0\}$. Then $f$ is strongly metricpreserving.

In the past two decades, a significant literature has developed on the subject of metricpreserving functions. The purpose of this paper is to introduce some of the results and techniques of the field to a broader mathematical audience.

We begin our study of metric-preserving functions in the next section, where we build tools to understand these functions and consider some revealing examples. Section 3 examines the important relationship between strongly metric-preserving functions and continuity. In the final section, we survey some of the results on differentiability in the context of metric-preserving functions. Many of the results discussed here have been garnered from papers that have appeared in other languages and in journals unfamiliar to the author; special thanks go to Jozef Doboš for his tremendous help in making so many of these papers available to me. Space constraints prevent me from discussing many avenues of research related to metric-preserving functions that have been pursued by various authors; see [8] for an excellent list of references. 
An interesting application of metric-preserving functions was discovered by Jůza in 1956, long before the subject had matured [13]. It is now well-known that there are complete nowhere discrete metric spaces that have a nested sequence of closed balls with empty intersection (of course the diameters of such balls cannot tend to 0). Jůza observed that the real line could be topologized to obtain such a space, using a metric-preserving function; in particular, he showed that $(\mathbf{R}, f \circ e)$ has the required property if $e$ is the usual metric on $\mathbf{R}$, and $f$ is the metric-preserving function defined by

$$
f(x)= \begin{cases}x & \text { if } x \leq 2 \\ 1+\frac{1}{x-1} & \text { if } x>2\end{cases}
$$

We prove that $f$ is metric-preserving in the next section; see [10] for technical refinements of this result.

2. Metric-Preserving Functions. A metric space is a set $X$ together with a function $d$ : $X \times X \rightarrow[0, \infty)$ satisfying the following three conditions:

(M1) For all $x, y \in X, d(x, y)=0$ if and only if $x=y$;

(M2) For all $x, y \in X, d(x, y)=d(y, x)$; and

(M3) For all $x, y, z \in X, d(x, y)+d(y, z) \geq d(x, z)$.

If a real-valued function $f$ defined on a set $S \subseteq \mathbf{R}$ is metric-preserving, then $S$ must include $[0, \infty)$, its range must lie in $[0, \infty)$, and $f^{-1}(0)=\{0\}$; we call such functions amenable. Since the values that an amenable function may have at negative reals have no bearing on whether the function is metric-preserving, we further require that all amenable functions have domain precisely $[0, \infty)$

The next proposition identifies a basic property of all metric-preserving functions:

Proposition 2.1. If $f$ is metric-preserving, then $f$ is subadditive.

Proof. Let $a, b \in[0, \infty)$ and let $d$ be the usual metric on $\mathbf{R}$. Then

$$
f(a)+f(b)=(f \circ d)(0, a)+(f \circ d)(a, a+b) \geq(f \circ d)(0, a+b)=f(a+b) .
$$

Terpe used the subadditivity criterion to show that a fairly broad class of functions is not metric-preserving. Before stating his result, we recall that a function $f:[0, \infty) \rightarrow[0, \infty)$ is convex 
on $[0, c]$ if

$$
f\left(\frac{x+y}{2}\right) \leq \frac{f(x)+f(y)}{2}=g\left(\frac{x+y}{2}\right)
$$

whenever $0 \leq x<y<z \leq c$, where the graph of $g$ is the line passing through $(x, f(x)),(y, f(y))$. Moreover, $f$ is strictly convex if $(2.1)$ holds when $\leq$ is replaced by $<$.

Corollary 2.2 [18]. Given $f:[0, \infty) \rightarrow[0, \infty)$, suppose that either

(A) $f$ is strictly convex on some interval including the origin and $f(0)=0$, or

(B) $f$ is differentiable on $(u, \infty)$ for some $u \geq 0$ and $\lim _{x \rightarrow \infty} f^{\prime}(x)=+\infty$.

Then $f$ is not metric-preserving.

Proof. For (A), let $c$ be a positive number for which $f$ is strictly convex on $[0, c]$. Then $f(c / 2)<$ $f(c) / 2$, whence $f(c / 2)+f(c / 2)<f(c)$, which violates subadditivity.

For (B), assume $f$ is differentiable on $(u, \infty), \lim _{x \rightarrow \infty} f^{\prime}(x)=+\infty$, and $f$ is metric-preserving. Let $x_{0}>u$. Because $f^{\prime}$ tends to $+\infty$, there is an $r>0$ such that for all $x>r, f^{\prime}(x)>f\left(x_{0}\right) / x_{0}$. Pick $x_{1}>r$ and use the Mean Value Theorem to obtain a $y \in\left(x_{1}, x_{1}+x_{0}\right)$ such that $f^{\prime}(y)=$ $\left(f\left(x_{1}+x_{0}\right)-f\left(x_{1}\right)\right) / x_{0}$. It follows that $\left(f\left(x_{1}+x_{0}\right)-f\left(x_{1}\right)\right) / x_{0}>f\left(x_{0}\right) / x_{0}$, which violates subadditivity.

Borsík and Doboš [1] give an example of a metric-preserving function $f$ that is differentiable on $(0, \infty)$ and satisfies $\limsup _{x \rightarrow \infty} f^{\prime}(x)=+\infty$, showing that the condition " $\lim _{x \rightarrow \infty} f^{\prime}(x)$ $=+\infty$ " in Corollary 2.2(B) is optimal. In the same paper, the authors extend Corollary 2.2(A); we state their result in Theorem 3.5. The proof makes use of a symmetry between subadditive and convex amenable functions, which is developed in the following exercise:

\section{Exercise 1 [1].}

(1) Suppose $f:[0, \infty) \rightarrow[0, \infty)$ is subadditive. Show that for all positive integers $n, f(n x) \leq n f(x)$ and $f\left(x / 2^{n}\right) \geq f(x) / 2^{n}$ whenever $x \geq 0$.

(2) Suppose $f$ is amenable and convex on $[0, c]$. Show that for all positive integers $n, f\left(x / 2^{n}\right) \leq$ $f(x) / 2^{n}$ whenever $0 \leq x \leq c$.

While subadditivity is an important necessary condition, the function $x /\left(1+x^{2}\right)$ shows that subadditivity is not sufficient for an amenable function to be metric-preserving. However, adding "nondecreasing" to subadditivity does yield a sufficient condition: 
Proposition 2.3. Suppose $f$ is amenable, subadditive, and nondecreasing. Then $f$ is metricpreserving.

Proof. Let $(X, d)$ be a metric space; we show $f \circ d$ is a metric. Properties (M1) and (M2) are easy to check. For (M3), let $x, y, z \in X$, and let $a=d(x, y), b=d(y, z)$, and $c=d(x, z)$. It suffices to show that $f(a)+f(b) \geq f(c)$. But

$$
\begin{aligned}
f(a)+f(b) & \geq f(a+b) \quad(\text { subadditive }) \\
& \geq f(c) \quad(\text { nondecreasing })
\end{aligned}
$$

as required.

Terpe [18] noticed that if $g:[0, \infty) \rightarrow[0, \infty)$ is non-increasing, then $\int_{0}^{x} g(t) d t$ is subadditive, and thus by Proposition 2.3, it is metric-preserving.

Another application of Proposition 2.3 involves concave functions: A function $f:[0, \infty) \rightarrow$ $[0, \infty)$ is concave if for all $x, y \geq 0$,

$$
f\left(\frac{x+y}{2}\right) \geq \frac{f(x)+f(y)}{2}
$$

Clearly, $f$ is concave if and only if $-f$ is convex on $[0, c]$ for every $c>0$. Ger and Kuczma [12] showed that concave amenable functions must be nondecreasing; since such functions are easily shown to be subadditive, we can use Proposition 2.3 again to conclude that they are also metric-preserving.

Exercise 2. Use either Proposition 2.3 or the fact that concave amenable functions are always metric-preserving to show that $\log _{a}(1+x)$, with $a>1$, and $x^{r}$, with $0<r \leq 1$, are metricpreserving.

Another interesting example of a metric-preserving function was discovered by Doboš [7] who showed that the extended Cantor function (extended to have value 1 for all $x>1$ ) is subadditive, and hence, by Proposition 2.3, is metric-preserving.

Our examples so far have been both nondecreasing and continuous. A simple example of a discontinuous nondecreasing metric-preserving function is

$$
f(x)= \begin{cases}0 & \text { if } x=0 \\ 1 & \text { otherwise }\end{cases}
$$


Proposition 2.3 ensures that this function is metric-preserving. It is also possible to obtain a continuous, metric-preserving function that fails to be nondecreasing. In order to construct this and other related examples, we need the notion of a triangle triplet, which is used to characterize metric-preserving functions. This notion first appeared in Sreenivasan's early paper [16], though the terminology itself did not appear in the literature until [2].

Definition 2.4. A triangle triplet is a triple $(a, b, c)$ of nonnegative reals for which $a \leq b+c, b \leq$ $a+c$, and $c \leq a+b$; equivalently, $|a-b| \leq c \leq a+b$.

Triangle triplets are precisely those triples of nonnegative reals that are of the form $(d(x, y), d(y, z), d(x, z))$ for some metric space $(X, d)$ and some $x, y, z \in X$. This observation follows from Proposition 2.5 and the proof of Proposition 2.6.

Proposition 2.5. If $(X, d)$ is a metric space and $x, y, z \in X$, then $(d(x, y), d(y, z), d(x, z))$ is a triangle triplet.

Proof. This is immediate from the triangle inequality.

Proposition 2.6 [2]. Suppose $f$ is amenable. Then the following are equivalent:

(1) $f$ is metric-preserving;

(2) for each triangle triplet $(a, b, c),(f(a), f(b), f(c))$ is a triangle triplet.

Proof of $(1) \Rightarrow(2)$. Given a triangle triplet $(a, b, c)$, let $d$ be the usual metric on $\mathbf{R}^{2}$. A straightforward argument using elementary geometry shows that there are $u, v, w \in \mathbf{R}^{2}$ such that $d(u, v)=a, d(v, w)=b$, and $d(u, w)=c$. The result now follows from Proposition 2.5.

Proof of $(2) \Rightarrow(1)$. Given $(X, d)$, we verify that $f \circ d$ is a metric. Properties (M1) and (M2) are immediate. For (M3), use (2) and the fact that $(d(x, y), d(y, z), d(x, z))$ is always a triangle triplet for $x, y, z \in X$.

For metric-preserving functions $f$, we obtain from Proposition 2.6(2) the inequality

$$
|f(a)-f(b)| \leq f(|a-b|)
$$

by letting $c=|a-b|$. 
Das [5] offers an alternative to Proposition 2.6(2) in his characterization of metric-preserving functions. A second alternative is the following:

Exercise 3([16], [18]). Show that Proposition 2.6(2) can be replaced by

$$
\text { for each triangle triplet }(a, b, c), f(a) \leq f(b)+f(c) \text {. }
$$

The proof of Proposition 2.6 shows that an amenable function $f$ is metric-preserving if and only if $f \circ d$ is a metric on $\mathbf{R}^{2}$ whenever $d$ is. Doboš gives an interesting example that shows $\mathbf{R}^{2}$ cannot be replaced by $\mathbf{R}$ [6]. He builds a variation $f$ of the extended Cantor function that preserves metrics on $\mathbf{R}$ and has the property that $\liminf _{x>0} f(x)=0$. The next proposition shows that such functions cannot be metric-preserving:

Proposition 2.7. Suppose $f$ is metric-preserving.

(1) For each $x_{0}>0$, there is an $\epsilon>0$ such that $f(x) \geq \epsilon$ for each $x \geq x_{0}$. In particular, $\lim \inf _{x>0} f(x)>0$, and $(x, 0)$ is not a limit point of the graph of $f$ for any $x>0$.

(2) If $f$ is discontinuous at 0 , there is some $\epsilon>0$ such that $f(x)>\epsilon$ for all $x>0$.

Proof of (1). If the assertion is false, there are $x_{0}>0$ and a sequence $\left\langle x_{n}\right\rangle_{n}$ such that $x_{n}>x_{0}$ for all $n$, and $\left\langle f\left(x_{n}\right)\right\rangle_{n} \longrightarrow 0$. Let $n$ be such that $f\left(x_{n}\right)<f\left(x_{0}\right) / 2$. Then $\left(x_{n}, x_{n}, x_{0}\right)$ is a triangle triplet, but $\left(f\left(x_{n}\right), f\left(x_{n}\right), f\left(x_{0}\right)\right)$ is not..

Proof of (2). If (2) is false, it follows from (1) that there is a decreasing sequence $\left\langle x_{n}\right\rangle_{n}$ such that $\left\langle x_{n}\right\rangle_{n} \longrightarrow 0$ and $\left\langle f\left(x_{n}\right)\right\rangle_{n} \longrightarrow 0$. By discontinuity at 0 , there are $\epsilon>0$ and a sequence $\left\langle y_{n}\right\rangle_{n}$ converging to 0 such that $f\left(y_{n}\right) \geq \epsilon$ for all $n$. Now let $n$ be such that $x_{n}<\epsilon / 2$ and let $m$ be such that $y_{m}<x_{n}$. Then $\left(x_{n}, x_{n}, y_{m}\right)$ is a triangle triplet, but $\left(f\left(x_{n}\right), f\left(x_{n}\right), f\left(y_{m}\right)\right)$ is not.

Proposition 2.7 shows that metric-preserving functions cannot have the $x$-axis as a horizontal asymptote; thus, the function $x /\left(1+x^{2}\right)$ is not metric-preserving.

Using Proposition 2.6, we can now exhibit quite a variety of discontinuous metric-preserving functions. We call an amenable function $f$ tightly bounded if there exists a $v>0$ such that $f(x) \in[v, 2 v]$ for all $x>0$.

Proposition 2.8 [2]. If $f$ is amenable and tightly bounded, then $f$ is metric-preserving. 
Proof. Let $v>0$ be such that $f(x) \in[v, 2 v]$ for all $x>0$, and let $(a, b, c)$ be a triangle triplet. Since the cases in which $a b c=0$ are trivial, we assume $a b c>0$. Then $f(a) \leq 2 v=v+v \leq f(b)+f(c)$, and Exercise 3 gives the desired conclusion.

Any amenable, tightly bounded function is necessarily discontinuous at 0 . It follows that there are $2^{\mathbf{c}}$ tightly bounded, amenable functions (where $\mathbf{c}$ is the cardinality of $\mathbf{R}$ ), so "most" metricpreserving functions are not continuous. A typical pathological example that one can construct with Proposition 2.8 is the following:

Proposition 2.9. There exists a metric-preserving function that is nowhere continuous and nowhere of bounded variation.

Proof. Let $\{A, B\}$ be a partition of $(0, \infty)$ such that both $A$ and $B$ are dense in $(0, \infty)$. Define $f:[0, \infty) \rightarrow[0, \infty)$ by

$$
f(x)= \begin{cases}0 & \text { if } x=0 \\ 1 & \text { if } x \in A \\ 2 & \text { if } x \in B\end{cases}
$$

Since $f$ is amenable and tightly bounded, it is metric-preserving; because of the choice of $A$ and $B, f$ has the required pathologies.

The example given in Proposition 2.9 shows, in particular, that metric-preserving functions need not be nondecreasing. Pokorný [15] has isolated a fairly natural class of amenable functions for which all metric-preserving functions must be nondecreasing: Define

$$
\mathcal{G}=\{g: \text { for some periodic } h:[0, \infty) \rightarrow[0, \infty), g(x)=x+h(x) \text { for all } x \geq 0\}
$$

An example of a member of $\mathcal{G}$ is $x+|\sin (x)|$. Pokorný showed that for amenable members $f$ of the class $\mathcal{G}, f$ is metric-preserving if and only if $f$ is nondecreasing and subadditive.

Proposition 2.9 also shows that metric-preserving functions need not be of bounded variation on any interval. Nonetheless, most of our examples of metric-preserving functions have this property. Terpe [17] formulated a sufficient condition, involving the notion of bounded gradient: Given $r>0$, we say that a metric-preserving function $f$ is of $r$-bounded gradient at 0 if there is some $h>0$ such that $f(x) \leq r x$ for all $x \in[0, h]$. And we say that $f$ is of bounded gradient at 0 if there is some $r>0$ such that $f$ is of $r$-bounded gradient at 0 . 
Proposition 2.10 [17]. If $f$ is metric-preserving and of bounded gradient at 0 , then $f$ is of bounded variation on each closed interval lying in $[0, \infty)$.

We postpone the proof of Proposition 2.10 until Section 4. There, we show that a metricpreserving function is of bounded gradient at 0 if and only if the derivative of $f$ at 0 exists and is finite. We are establishing a global property of $f$ (namely, bounded variation on each closed interval) based on the behavior of $f$ at 0 . This theme reappears when we consider continuity in the next section.

For the remainder of this section, we discuss techniques for building new metric-preserving functions from old and apply these to answer several natural questions:

Q1. Can a metric-preserving function be strictly decreasing on an interval $(a, \infty), a \geq 0$ ?

Q2. Can a continuous metric-preserving function be strictly decreasing on an interval $(a, \infty)$, $a \geq 0$ ?

Q3. Must every continuous, nondecreasing metric-preserving function be concave?

Q4. Must every discontinuous metric-preserving function be tightly bounded?

Q5. Must every metric-preserving function that is continuous at 0 be continuous?

To answer Question Q1, we first show that every bounded function $[0, \infty) \rightarrow[0, \infty)$ has an upward translation that is tightly bounded. For each $f:[0, \infty) \rightarrow[0, \infty)$ and each $r>0$ we define

$$
U_{f, r}(x)= \begin{cases}0, & \text { if } x=0 \\ f(x)+r, & \text { if } x>0\end{cases}
$$

Proposition 2.11. Suppose $f:[0, \infty) \rightarrow[0, \infty)$ is bounded above. Then there is an $r_{0}>0$ such that $U_{f, r}$ is metric-preserving for all $r \geq r_{0}$.

Proof. Let $r_{0}$ be an upper bound for $f$ and note that $U_{f, r}$ is tightly bounded for all $r \geq r_{0}$.

Example 2.12. A metric-preserving function that is strictly decreasing on $(0, \infty)$. Define

$$
g(x)= \begin{cases}0 & \text { if } x=0 \\ 1+\frac{1}{x+1} & \text { if } x>0\end{cases}
$$

Now, $g=U_{f, 1}$ where

$$
f(x)= \begin{cases}0 & \text { if } x=0 \\ \frac{1}{x+1} & \text { if } x>0\end{cases}
$$


By Proposition 2.11, $g$ is metric-preserving and is decreasing on $(0, \infty)$. This example answers Question Q1.

The next proposition provides a tool for answering Questions Q2 and Q3.

Proposition 2.13 [2, Proposition 2.16]. Suppose $f$ is metric-preserving and suppose $c, r>0$. Define $T_{f, c, r}:[0, \infty) \rightarrow[0, \infty)$ by

$$
T_{f, c, r}(x)= \begin{cases}r x & \text { if } x \in[0, c) \\ f(x) & \text { otherwise }\end{cases}
$$

Then $T_{f, c, r}$ is metric-preserving if and only if $f(c)=r c$ and $|f(x)-f(y)| \leq r|x-y|$ for all $x, y \in[c, \infty)$.

In [11], the authors generalize this result, replacing the function $r x$ in the defintion of $T_{f, c, r}$ by a concave metric-preserving function $g:[0, \infty) \rightarrow[0, \infty)$. Let $T_{f, g, c}$ denote the function defined from $f$ and $g$ in this way. As the authors of [11] show, if $|x-y| \leq c$ implies $|f(x)-f(y)| \leq g(|x-y|)$ for all $x, y \geq c$, then $T_{f, g, c}$ is metric-preserving.

Example 2.14. A metric-preserving, continuous function that is strictly decreasing on $(1, \infty)$. Let $g$ be as in Example 2.12. Define

$$
T(x)= \begin{cases}\frac{3}{2} x & \text { if } x \in[0,1] \\ g(x) & \text { otherwise }\end{cases}
$$

Clearly, $T$ is continuous and strictly decreasing on $(1, \infty)$. Since $T=T_{g, 1,1}$, Proposition 2.13 ensures that $T$ is metric-preserving. This example settles Question Q2. It also shows that continuous metric-preserving functions need not be nondecreasing.

Example 2.15 [18]. A continuous, nondecreasing, metric-preserving function that is not concave.

Define

$$
f(x)= \begin{cases}1 & \text { if } 0 \leq x<2 \\ x-1 & \text { if } 2 \leq x<3 \\ 2 & \text { otherwise }\end{cases}
$$

Since $f$ is tightly bounded, $f$ is metric-preserving. Define

$$
T(x)= \begin{cases}x & \text { if } x \in[0,1] \\ f(x) & \text { otherwise. }\end{cases}
$$

Clearly $T$ is continuous and nondecreasing; $T$ is not concave since

$$
T\left(\frac{a+b}{2}\right)<\frac{T(a)+T(b)}{2}
$$


when $a=1$ and $b=3$. Since $T=T_{f, 1,1}$, Proposition 2.13 can be applied to show $T$ is metricpreserving. This example settles Question Q3.

Exercise 4 [13]. Use Propositions 2.11 and Proposition 2.13 to show that Jüa's function (1.1) is metric-preserving.

To answer Question Q4, we invoke one of the closure properties of the class of metric-preserving functions. We summarize these in the following theorem; we omit the straightforward proofs.

Theorem 2.16 [1], [2], [17].

(1) If $f, g$ are metric-preserving and $m>0$, then each of $f \circ g, f+g, m f$ and $\max (f, g)$ is metricpreserving.

(2) If $\left\langle h_{n}\right\rangle_{n}$ are metric-preserving functions that converge pointwise to a function $h$ and $h(x)>0$ for all $x$, then $h$ is metric-preserving. Likewise, if $\sum_{n=1}^{\infty} h_{n}$ converges to a function $\bar{h}$, where each function $h_{n}$ is metric-preserving, then $\bar{h}$ is metric-preserving.

(3) If $S$ is any set of metric-preserving functions that is pointwise bounded and if we define $g(x)=\sup \{f(x): f \in S\}$, then $g$ is metric-preserving.

Example 5 [2]. A discontinuous and metric-preserving function that is not tightly bounded. Define

$$
f(x)= \begin{cases}0 & \text { if } x=0 \\ 1+|x-1| & \text { otherwise }\end{cases}
$$

The function $f$ is discontinuous at 0 and not tightly bounded. Now, $f=\max (g, h)$, where $g(x)=x$ and

$$
h(x)= \begin{cases}0 & \text { if } x=0 \\ 1+|x-1| & \text { if } x \in(0,2) \\ 2 & \text { otherwise. }\end{cases}
$$

Since $h$ is metric-preserving (because it is tightly bounded), and $g$ is metric-preserving, it follows from Theorem 2.16(1) that $f$ is metric-preserving as well. This example settles Question Q4.

We postpone a discussion of Question Q5 until the next section, where it is a central topic.

\section{Strongly Metric-Preserving Functions}

In this section we characterize the metric-preserving functions that are strongly metricpreserving. An important theme here is the significance of the behavior of a metric-preserving 
function at 0 : We show that such an $f$ is strongly metric-preserving if and only if it is continuous at 0 .

We begin with some notation and an observation. For a metric space $(X, d), x \in X, \epsilon>0$, write:

$$
N(d, x, \epsilon)=\{y \in X: d(x, y)<\epsilon\} .
$$

Lemma 3.1. Suppose $f$ is metric-preserving. Then the following are equivalent:

(1) $f$ is discontinuous at 0 ;

(2) $f \circ d$ is a discrete metric for every metric $d$.

Proof of $(1) \Rightarrow(2)$. Let $(X, d)$ be a metric space. By Proposition 2.7, there is an $\epsilon>0$ such that $f(z)>\epsilon$ for all $z>0$. Then $N(f \circ d, x, \epsilon)=\{x\}$ for each $x \in X$, as required.

Proof of $(2) \Rightarrow(1)$. Let $d$ be the usual metric on $\mathbf{R}$ and let $\epsilon>0$ be such that $N(f \circ d, 0, \epsilon)=\{0\}$. It follows that there is a sequence $\left\langle x_{n}\right\rangle_{n}$ of positive numbers converging to 0 (relative to $d$ ) such that $\epsilon<f\left(d\left(x_{n}, 0\right)\right)=f\left(x_{n}\right)$ for all $n$. This establishes (1).

The next theorem first appeared in [2]; one direction of the theorem was observed in [16].

Theorem 3.2 [2]. A metric-preserving function is strongly metric-preserving if and only if it is continuous at 0 .

Proof. One direction follows from Lemma 3.1. For the other direction, suppose $f$ is continuous at 0 and metric-preserving. Let $(X, d)$ be a metric space. We show that $f \circ d$ and $d$ are equivalent metrics. Let $\epsilon>0$ and $x \in X$. By continuity, let $\delta \leq \epsilon$ be such that $f(z)<\epsilon$ whenever $0 \leq z<\delta$. Then $N(d, x, \delta) \subseteq N(f \circ d, x, \epsilon)$. Hence, the $d$-topology refines the $f \circ d$-topology. To show the converse, again start with $x \in X$ and let $r>0$. Use Proposition 2.7(1) to obtain an $\epsilon>0$ such that $f(z) \geq \epsilon$ for all $z \geq r$. But now $N(f \circ d, x, \epsilon) \subseteq N(d, x, r)$, and we are done.

In [2], the authors develop Theorem 3.2 further by providing necessary and sufficient conditions on a metric-preserving function $f$ for $d$ and $f \circ d$ to be uniformly equivalent as well.

As we now show, continuity at 0 forces a metric-preserving function to be continuous everywhere; this result answers Question Q5 from Section 2. 
Theorem 3.3 [2]. Suppose $f$ is metric-preserving and continuous at 0 . Then $f$ is continuous on $[0, \infty)$.

Proof. Assume that $f$ is not continuous at some $x_{0}>0$. Let $\epsilon>0$ be such that there are $z$ arbitrarily close to $x_{0}$ for which $\left|f(z)-f\left(x_{0}\right)\right| \geq \epsilon$. By continuity at 0 , let $\delta<x_{0} / 2$ be such that $0 \leq a<\delta$ implies $f(a)<\epsilon$. Now pick $z_{0}$ so that $\left|z_{0}-x_{0}\right|<\delta$ and $\left|f\left(z_{0}\right)-f\left(x_{0}\right)\right| \geq \epsilon$. Let $a_{0}=\left|z_{0}-x_{0}\right|$.

Case 1. $f\left(z_{0}\right) \geq f\left(x_{0}\right)+\epsilon$.

If $x_{0}+a_{0}=z_{0}$, then $f\left(x_{0}\right)+f\left(a_{0}\right)<f\left(x_{0}\right)+\epsilon \leq f\left(x_{0}+a_{0}\right)$, which violates subadditivity.

On the other hand, if $z_{0}+a_{0}=x_{0}$, notice that $\left(x_{0}, z_{0}, a_{0}\right)$ is a triangle triplet, and in particular that $x_{0}-z_{0}=a_{0}<\delta$. But since $f\left(z_{0}\right)-f\left(x_{0}\right) \geq \epsilon$ and $f\left(a_{0}\right)<\epsilon,\left(f\left(x_{0}\right), f\left(z_{0}\right), f\left(a_{0}\right)\right)$ is not a triangle triplet, which violates Proposition 2.3.

Case 2. $f\left(x_{0}\right) \geq f\left(z_{0}\right)+\epsilon$.

The proof in this case is similar to that in Case 1, and we omit it.

In each case, we have obtained a contradiction from the assumption that $x_{0}$ is a point of discontinuity, as required.

Thus, for a metric-preserving function $f$, the global properties of continuity and being strongly metric-preserving are completely determined by the behavior of $f$ at 0 . And continuity of $f$ at 0 is determined by a property that is apparently even weaker: It follows from Proposition 2.7(2) that $f$ is continuous at 0 if and only if, for each $\epsilon>0$, there is an $x>0$ with $f(x)<\epsilon$. We have proved the following:

Theorem 3.4 [2]. Suppose $f$ is metric-preserving. Then the following are equivalent:

(1) $f$ is strongly metric-preserving;

(2) $f$ is continuous at 0 ;

(3) $f$ is continuous on $[0, \infty)$;

(4) for each $\epsilon>0$, there is an $x>0$ with $f(x)<\epsilon$.

As an application of Theorem 3.4, we give a proof of a result in [1] that improves upon Corollary 2.2(A): 
Theorem 3.5. If $f$ is metric-preserving and is convex on $[0, c]$ for some $c>0$, then $f$ is linear on $[0, c]$.

Proof. Let $f$ be metric-preserving and convex on $[0, c]$. We establish the conclusion of the theorem from the following three claims:

Claim 1. For each $x \in[0, c]$ and each positive integer $n, f\left(x / 2^{n}\right)=f(x) / 2^{n}$.

Claim 2. The function $f$ is continuous.

Claim 3. Whenever $0<x \leq y \leq c, f(x) / x \leq f(y) / y$.

Using these claims, we prove the result by showing that $f(x)=(f(c) / c) x$ for all $x \in[0, c]$. Since this relation is obvious for $x=0$, let $x \in(0, c]$, and let $n$ be such that $c / 2^{n} \leq x$. Then by Claims 1 and 3,

$$
\frac{f(c)}{c}=\frac{f\left(\frac{c}{2^{n}}\right)}{\frac{c}{2^{n}}} \leq \frac{f(x)}{x} \leq \frac{f(c)}{c},
$$

as required.

We turn to the proofs of the claims. Claim 1 is proved by combining parts (1) and (2) of Exercise 1. For Claim 2, we use Claim 1 and Theorem 3.4(4): For any $\epsilon>0$, we obtain $x$ for which $f(x)<\epsilon$ by setting $x=\epsilon / 2^{n}$, where $n$ is such that $f(\epsilon) / 2^{n}<\epsilon$.

To prove Claim 3, let $A=\{u \in[0, c]: f(x) / x \leq f(y) / y$ whenever $0<x \leq y \leq u\}$. Vacuously, $A$ is nonempty. Let $z=\sup A$; we prove $A=[0, c]$ by showing $z=c$. Seeking a contradiction, assume $z<c$. There are two cases to consider:

Case 1: $z \notin A$. From our assumptions, it follows that there is an $x_{0}<z$ such that $f(x) / x>f(z) / z$ whenever $x_{0} \leq x<z$. For each $\operatorname{such} x$, let $g_{x}:[0, \infty) \rightarrow[0, \infty)$ denote the function whose graph is the line through $(0,0)$ and $(x, f(x))$. By the choice of $z$, whenever $x_{0} \leq x \leq y \leq z$, we have $g_{x_{0}}(z)>f(z)$ and $g_{x}(y) \geq g_{x_{0}}(y)$. By continuity of $g_{x_{0}}$, there is a $\delta$ such that $0<\delta \leq z-x_{0}$ and $g_{x_{0}}(x)>f(z)$ whenever $0<z-x \leq \delta$. Let $x_{1}=z-\delta$, and let $\epsilon=g_{x_{0}}\left(x_{1}\right)-f(z)$. Then, whenever $0<z-x \leq \delta$

$$
|f(x)-f(z)|=g_{x}(x)-f(z) \geq g_{x_{0}}(x)-f(z) \geq g_{x_{0}}\left(x_{1}\right)-f(z)=\epsilon,
$$

which contradicts the continuity of $f$ at $z$. 
Case 2: $z \in A$. By our assumptions, we can find $y$ such that $f(z) / z>f(y) / y$ where $y>z$ and $y$ is arbitrarily close to $z$. Pick such a $y$ with $y-z<z$. Set $x=2 z-y$. Then $z=(x+y) / 2$, and by a straightforward computation,

$$
\frac{f(z)-f(x)}{z-x} \leq \frac{f(y)-f(z)}{y-z}
$$

However, it is easy to verify that

$$
\frac{f(x)}{x} \leq \frac{f(z)}{z} \text { implies } \frac{f(z)}{z} \leq \frac{f(z)-f(x)}{z-x}
$$

and

$$
\frac{f(y)}{y}<\frac{f(z)}{z} \text { implies } \frac{f(y)-f(z)}{y-z}<\frac{f(z)}{z} .
$$

Combining the conclusions of (3.2) and (3.3), we have

$$
\frac{f(z)-f(x)}{z-x}>\frac{f(y)-f(z)}{y-z}
$$

which contradicts (3.1). This completes the proof of Claim 3 and the theorem.

Metric-preserving functions can be viewed as a tool for producing new metrics of the form $f \circ d$ on a given metric space $(X, d)$. The results of this section show that the usefulness of this tool does not lie in generating topologically distinct metrics; indeed, at most two distinct metrics, up to topological equivalence, can be obtained in this way: the discrete metric and $d$ itself. More useful is the fact that whenever $X$ has cardinality at least 2, there are $\mathbf{c}$ distinct metrics on $X$ of the form $f \circ d$, where $f$ is metric-preserving. This fact suggests that the class of metric-preserving functions may be a rich source for constructing metrics that are topologically equivalent to $d$ but that exhibit new, mathematically interesting properties. Indeed, Jůza's function (1.1) is just this kind of example.

\section{Metric-Preserving Functions and Differentiability}

Mirroring the situation for continuity, the notion of differentiability partitions the class of metric-preserving functions into two rather different subclasses determined by the value of the 
derivative of each function at 0 . We shall see that the (extended) derivative of such a function always exists at 0 ; the central question is whether the derivative is finite or infinite. Functions with finite derivative form a well-behaved class of continuous functions that are differentiable almost everywhere; functions with infinite derivative, by contrast, can be very unruly - they can be continuous, nowhere differentiable (in the finite sense), and even, as we saw in Section 2, nowhere continuous. In this section we outline proofs of these results, which we have extracted from [1].

We first show that for any metric-preserving function $f, f^{\prime}(0)$ exists in the extended sense. The proof naturally divides into two parts depending on whether the set $K_{f}=\{r>0: f(x) \leq$ $r x$ for all $x \geq 0\}$ is empty. In the course of the proof, we show that $f^{\prime}(0)<+\infty$ if and only if $K_{f} \neq \emptyset$, and $f^{\prime}(0)=+\infty$ if and only if $K_{f}=\emptyset$. The next lemma is the first step in proving the result for the latter case.

Lemma 4.1 [1]. If $f$ is metric-preserving and $0<x \leq y$, then $f(y) / y \leq 2 f(x) / x$.

Proof. Let $n$ be a positive integer such that

$$
2^{n-1} \leq \frac{y}{x}<2^{n}
$$

Then $\left(x, x, y / 2^{n-1}\right)$ is a triangle triplet since $y / 2^{n-1}<2 x$. Thus, $f(y) / 2^{n-1} \leq f\left(y / 2^{n-1}\right) \leq 2 f(x)$, where the first inequality follows from Exercise 1(1). Hence, using (4.1), $f(y) \leq 2^{n-1}(2 f(x)) \leq$ $2 y f(x) / x$, and the result follows.

We can now prove that $f^{\prime}(0)$ exists and is infinite when $K_{f}=\emptyset$ : Let $n$ be a positive integer. Since $K_{f}=\emptyset$, we can pick $y>0$ such that $f(y) \geq 2 n y$. Let $x \in(0, y]$. By Lemma $4.1,2 n \leq$ $f(y) / y \leq 2 f(x) / x$. But now we have shown that for each integer $n>0$, there is $y>0$ such that $f(x) / x \geq n$ whenever $0<x \leq y$, as required.

We turn to the case in which $K_{f} \neq \emptyset$. Suppose $f$ is metric-preserving and that there is an $h>0$ such that $f(x) \leq r x$ for all $x \in[0, h]$ (that is, $f$ is of $r$-bounded gradient at 0 , as in Proposition 2.10). We show that in fact, $f(x) \leq r x$ for all $x \geq 0$. To see this, let $x \geq 0$ and let $n$ be a large enough integer so that $x / 2 n \leq h$. By Exercise $1(1), f(x) / 2^{n} \leq f\left(x / 2^{n}\right) \leq r x / 2^{n}$, whence, $f(x) \leq r x$. Combining this result with $(2.2)$, we also have $|f(x)-f(y)| \leq r|x-y|$ for all $x, y \geq 0$. Thus: 
Proposition 4.2 [1]. Suppose $r>0$ and $f$ is metric-preserving and of $r$-bounded gradient at 0.

Then,

(1) $f(x) \leq r x$ for all $x \geq 0$;

(2) $|f(x)-f(y)| \leq r|x-y|$ for all $x, y \geq 0$.

The next result that we need is a generalization of Theorem 3.5:

Lemma 4.3 [1]. Suppose $f$ is metric-preserving and $r>0$. If in every neighborhood of 0 there is a point a such that $f(a)=r a$, then there is an $h>0$ such that $f(x)=r x$ for all $x \in[0, h]$.

Proof. The hypothesis and Theorem 3.4(4) imply that $f$ is continuous. Let $h>0$ be such that $f(h)=r h$. Assume there is $x \in(0, h)$ with $f(x) \neq x$. Let $A=\{y \in[0, \infty): f(y)=r y\}$. By continuity of $f, A \cap[0, x]$ is compact and so has a maximum element, which we denote by $m_{0, x}$; likewise, $A \cap[x, h]$ is compact, and we denote its minimum element by $m_{x, h}$.

If $f(x)>r x$, we show there is a number $u \in\left(m_{0, x}, x\right)$ such that $f(u) \leq r u$, from which it follows, by continuity, that $f(z)=r z$ for some $z \in[u, x)$, contradicting the choice of $m_{0, x}$. To obtain $u$, let $y \in A$ with $0<y<x-m_{0, x}$. Now let $u=y+m_{0, x}$ and observe that

$$
f\left(y+m_{0, x}\right) \leq f(y)+f\left(m_{0, x}\right)=r y+r m_{0, x}=r\left(y+m_{0, x}\right) .
$$

Similarly, if $f(x)<r x$, one obtains a $u \in\left(x, m_{x, h}\right)$ such that $f(u) \geq r u$, yielding a contradiction, as before. Here, pick $z \in A$ with $0<z<m_{x, h}-x$ and set $u=m_{x, h}-z$. We leave to the reader the verification that $u$ has the required property.

Now we prove that $f^{\prime}(0)$ exists and is finite in the case $K_{f} \neq \emptyset$. By Theorem 3.4, $f$ is continuous, whence $K_{f}$ is closed. Let $r_{0}=\min K_{f}$. We show

$$
r_{0}=\lim _{x \rightarrow 0} \frac{f(x)}{x}
$$

Let $\epsilon>0$. We claim that:

$$
\text { for each } h>0 \text {, there is an } x \in(0, h] \text { such that } f(x)>\left(r_{0}-\epsilon\right) x \text {. }
$$


To see this, assume instead that there is an $h>0$ such that for all $x \in[0, h], f(x) \leq r_{1} x$, where $r_{1}=r_{0}-\epsilon$. Thus, $f$ is of $r_{1}$-bounded gradient at 0 , and so by Proposition 4.2(1), $f(x) \leq r_{1} x$ for all $x \geq 0$, which contradicts the choice of $r_{0}$.

Next, we show that

there exists an $h>0$ such that $f(x)>\left(r_{0}-\epsilon\right) x$ for all $x \in(0, h]$.

Assume instead that

$$
\text { for each } h>0 \text {, there exists an } x \in[0, h] \text { such that } f(x) \leq\left(r_{0}-\epsilon\right) x \text {. }
$$

Let $h>0$. By (4.3), there is an $x_{1} \in(0, h]$ such that $f\left(x_{1}\right)>\left(r_{0}-\epsilon\right) x_{1}$, and by (4.5), there is an $x_{2} \in(0, h]$ such that $f\left(x_{2}\right) \leq\left(r_{0}-\epsilon\right) x_{2}$. By the continuity of $f$, there is an $x_{3} \in(0, h]$ such that $f\left(x_{3}\right)=\left(r_{0}-\epsilon\right) x_{3}$. By Lemma 4.3, $f(x)=\left(r_{0}-\epsilon\right) x$ holds on some neighborhood of 0 , contradicting (4.3). This proves (4.4) .

Now since $r_{0} \in K_{f}$, we also have $f(x)<\left(r_{0}+\epsilon\right) x$ for each $x>0$. Thus, combining these results, we obtain: for each $\epsilon>0$, there exists an $h>0$ such that $r_{0}-\epsilon<f(x) / x<r_{0}+\epsilon$ whenever $0<x \leq h$; that is, (4.2) holds, as required.

We have proved:

Theorem 4.4 [1]. Let $f$ be a metric-preserving function. Then $f^{\prime}(0)$ exists (in the extended sense).

We now show that a metric-preserving function $f$ with finite derivative at 0 must be differentiable almost everywhere. We begin with a key lemma:

Lemma 4.5 [1]. Suppose $f$ is metric-preserving and $f^{\prime}(0)<+\infty$. Then

(1) $f(x) \leq f^{\prime}(0)$ for all $x \geq 0$;

(2) $|f(x)-f(y)| \leq f^{\prime}(0)|x-y|$ for all $x, y \geq 0$.

Proof. To prove (1), let $\epsilon>0$. Since $f^{\prime}(0)<+\infty$, there is an $h>0$ such that

$$
f(x) \leq\left(f^{\prime}(0)+\epsilon\right) x
$$


for all $x \in[0, h]$; that is, $f$ is of $\left(f^{\prime}(0)+\epsilon\right)$-bounded gradient at 0. By Proposition 4.2, (4.6) holds for all $x \geq 0$. Since $\epsilon>0$ was arbitrary, the result follows. Part (2) can be proved by again applying (2.2).

The proof of Lemma 4.5(1) shows that if $f$ is metric-preserving and $f^{\prime}(0)<+\infty$, then $f$ is of bounded gradient at 0 . The converse is also true, and follows immediately from Theorem 4.4. Thus:

Corollary 4.6. Let $f$ be a metric-preserving function. Then $f^{\prime}(0)<+\infty$ if and only if $f$ is of bounded gradient at 0 .

Our target theorem falls out directly from Lemma 4.5(2) and establishes Proposition 2.10:

Theorem 4.7 [1]. Suppose $f$ is metric-preserving and $f^{\prime}(0)<+\infty$. Then $f$ is of bounded variation on each closed interval lying in $[0, \infty)$. Hence, $f$ is differentiable almost everywhere.

Finally, we consider the subclass of metric-preserving functions $f$ for which $f^{\prime}(0)=+\infty$. The following example, due to Doboš and Piotrowski, is a slight modification of Van der Waerden's continuous nowhere differentiable function [3].

Example 4.8 [11]. A metric-preserving function that is continuous and nowhere differentiable. Define $h:[0, \infty) \rightarrow[0, \infty)$ by

$$
h(x)= \begin{cases}x & \text { if } x \leq \frac{1}{2} \\ \frac{1}{2}+\left|x-[x]-\frac{1}{2}\right| & \text { if } x>\frac{1}{2}\end{cases}
$$

where $[a]$ denotes the integer part of $a$. It is easy to verify that $h=T_{g, \frac{1}{2}, 1}$ for some tightly bounded function $g$ (recall Proposition 2.13), and so $h$ is metric-preserving. Likewise, for each integer $n \geq 0$, $h\left(2^{n} \cdot x\right)$ is metric-preserving, and using Theorem $4.9(1)$, so is $h\left(2^{n} \cdot x\right) / 2^{n}$. Theorem $4.10(2)$ ensures that

$$
f(x)=\sum_{n=0}^{\infty} \frac{h\left(2^{n} \cdot x\right)}{2^{n}}
$$

is metric-preserving as well. The proof that $f$ is continuous and nowhere differentiable is essentially the same as Van der Waerden's.

The extent to which the class of metric-preserving functions is structured around their behavior at 0 has been a striking theme in this brief survey. Whether a metric-preserving function $f$ 
transforms each metric $d$ to a discrete metric or to a metric that is topologically equivalent to $d$ is determined by whether $f$ is continuous at 0 . And whether $f$ has the property of being (finitely) differentiable almost everywhere but nowhere infinitely differentiable is determined by whether $f^{\prime}(0)$ is finite or infinite. Although the results presented here offer many possible directions for generalization and further study, one of the most compelling is this:

Which behaviors of a metric-preserving function $f$ at 0 - such as continuity at 0 or " $f^{\prime}(0)<+\infty$ " - determine interesting global properties of $f$ ?

\section{REFERENCES}

1. J. Borsík and J. Doboš, On metric preserving functions, Real Anal. Exchange 13 (1987-88) $285-293$.

2. _ Functions whose composition with every metric is a metric, Math. Slovaca 31 (1981) 3-12 (in Russian).

3. P. Billingsley, Van der Waerden's continuous nowhere differentiable function, Amer. Math. Monthly 89 (1982) 691.

4. A. Bruckner, Differentiation of Real Functions, Lecture Notes In Mathematics 659, SpringerVerlag, New York, 1978.

5. P. P. Das, Metricity preserving transforms, Pattern Recognition Letters 10 (1989) 73-76.

6. J. Doboš, On modifications of the Euclidean metric on reals, Tatra Mt. Math. Publ. 8 (1996) 51-54.

7. 3425-6.

8. _ A survey of metric-preserving functions, Questions Answers Gen. Topology 13 (1995) 129-133.

9. J. Doboš, Z. Piotrowski, When distance means money, Internat. J. Math. Ed. Sci. Tech. 28 (1997) 513-518.

10. — A note on metric-preserving functions, Internat. J. Math. Math. Sci. 19 (1996) 199-200. 
11. Some remarks on metric-preserving functions, Real Anal. Exchange 19 (1993-94) $317-320$.

12. R. Ger and M. Kuczma, On the boundedness and continuity of convex functions and additive functions, Aequationes Math. 4 (1970) 157-162

13. M. Jůza, A note on complete metric spaces, Matematicko-fyzikálny časopis SAV 6 (1956) 143-148 (in Czech)

14. J. Kelley, General Topology, Springer-Verlag, New York, 1955.

15. I. Pokorný, Some remarks on metric preserving functions, Tatra Mt. Math. Publ. 2 (1993) $65-68$.

16. T. K. Sreenivasan, Some properties of distance functions, J. Indian Math. Soc. (N.S.) 11 (1947) 38-43.

17. F. Terpe, Some properties of metric preserving functions, Proc. Conf. Topology, Measure and Fractals, Math. Res. 66, Akademie-Verlag, Berlin, 1992, pp. 214-217.

18. _ Metric preserving functions, Proc. Conf. Topology and Measure IV, Greifswald, 1984, pp. 189-197.

19. W. A. Wilson, On certain types of continuous transformations of metric spaces, Amer. J. Math. 57 (1935) 62-68.

Maharishi University of Management, c/o 310 E. Washington Ave., Fairfield, IA 52556

pcorazza@kdsi.net 